\title{
Hydrodynamical simulations of the barred spiral galaxy NGC 1097
}

\author{
Lien-Hsuan Lin ${ }^{1}$, Hsiang-Hsu Wang, Pei-Ying Hsieh, \\ Ronald E. Taam, Chao-Chin Yang and David C. C. Yen
}

\author{
${ }^{1}$ Institute of Astronomy and Astrophysics, Academia Sinica, P.O. Box 23-141, Taipei 10617, \\ Taiwan, R.O.C. \\ email: lhlin@asiaa.sinica.edu.tw
}

\begin{abstract}
NGC 1097 is a nearby barred spiral galaxy believed to be interacting with the elliptical galaxy NGC 1097A located to its northwest. It hosts a Seyfert 1 nucleus surrounded by a circumnuclear starburst ring. Two straight dust lanes connected to the ring extend almost continuously out to the bar. The other ends of the dust lanes attach to two main spiral arms. To provide a physical understanding of its structural and kinematical properties, two-dimensional hydrodynamical simulations have been carried out. Numerical calculations reveal that many features of the gas morphology and kinematics can be reproduced provided that the gas flow is governed by a gravitational potential associated with a slowly rotating strong bar. By including the self-gravity of the gas disk in our calculation, we have found the starburst ring to be gravitationally unstable which is consistent with the observation in Hsieh et al. (2011). Our simulations also show that gas can flow into the region within the starburst ring even after its formation, leading to the coexistence of both a nuclear ring and a circumnuclear disk.
\end{abstract}

Keywords. hydrodynamics, accretion, instabilities, galaxies: individual(NGC 1097) - galaxies: evolution - galaxies: spiral — galaxies: structure
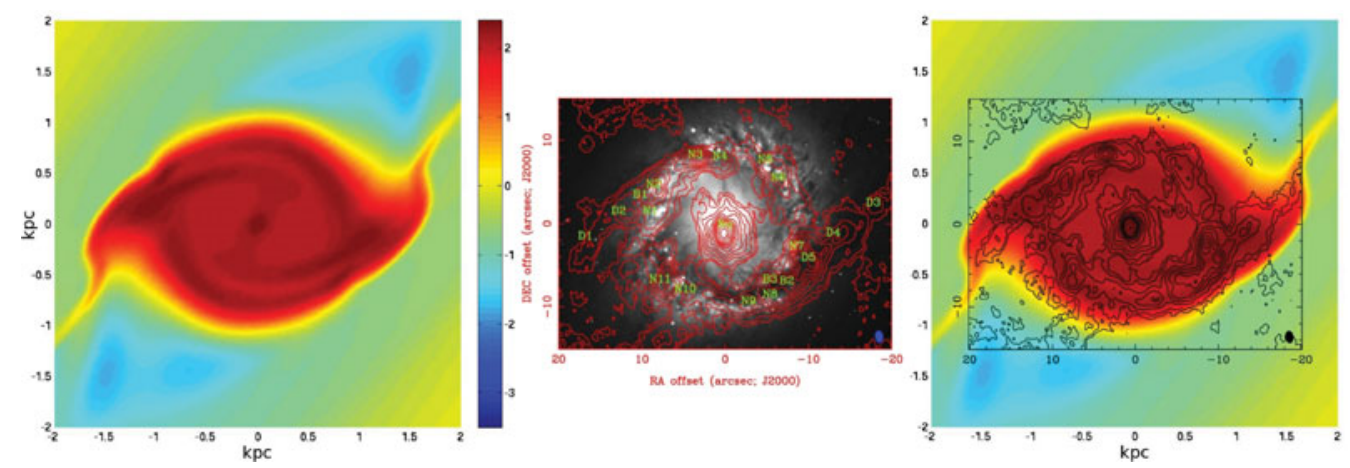

Figure 1. Left: The central part of the projected simulated density map. Middle: ${ }^{12} \mathrm{CO}(2-1)$-integrated map (contours) overlaid on the HSTI-band image (grayscale) (Fig. 4 in Hsieh et al. 2011). Right: The superposition of the left panel and the ${ }^{12} \mathrm{CO}(2-1)$ emission (contours). The starburst ring in the simulation is of the same size as that in the observation. The two groups of strongest emission knots in the northeast and the southwest parts of the starburst ring in the observation coincide with the densest regions in the simulation.

\section{Reference}

Hsieh, Pei-Ying, Matsushita, Satoki, Liu, Guilin, Ho, Paul T. P., Oi \& Nagisa; Wu, Ya-Lin 2011, ApJ 736, 129 